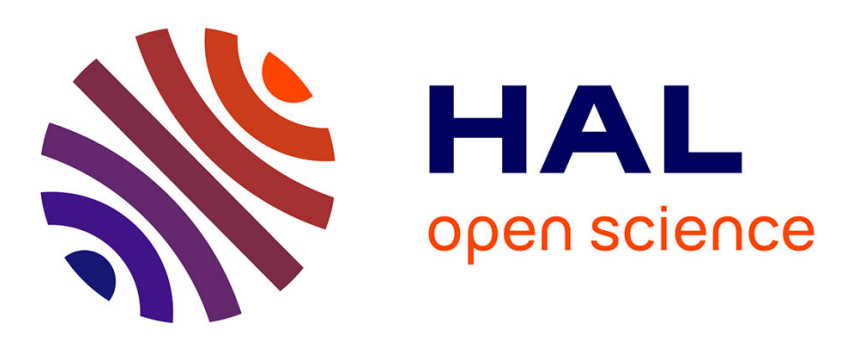

\title{
WhatsOpt: a web application for multidisciplinary design analysis and optimization
}

Rémi Lafage, Sebastien Defoort, Thierry Lefebvre

\section{To cite this version:}

Rémi Lafage, Sebastien Defoort, Thierry Lefebvre. WhatsOpt: a web application for multidisciplinary design analysis and optimization. AIAA Aviation 2019 Forum, Jun 2019, Dallas, United States. 10.2514/6.2019-2990 . hal-03010165

\section{HAL Id: hal-03010165 \\ https://hal.science/hal-03010165}

Submitted on 1 Dec 2020

HAL is a multi-disciplinary open access archive for the deposit and dissemination of scientific research documents, whether they are published or not. The documents may come from teaching and research institutions in France or abroad, or from public or private research centers.
L'archive ouverte pluridisciplinaire HAL, est destinée au dépôt et à la diffusion de documents scientifiques de niveau recherche, publiés ou non, émanant des établissements d'enseignement et de recherche français ou étrangers, des laboratoires publics ou privés. 


\title{
WhatsOpt: a web application for multidisciplinary design analysis and optimization
}

\author{
Rémi Lafage*, Sebastien Defoort ${ }^{\dagger}$, Thierry Lefèbvre \\ ONERA/DTIS, Université de Toulouse, Toulouse, France
}

\begin{abstract}
This paper describes ONERA's on-going effort to develop a collaborative environment to support multidisciplinary design analysis and optimization in the context of overall vehicle design team activities. This environment, namely WhatsOpt, is a web application allowing the ONERA experts to define collaboratively aircraft multi-disciplinary analyses in terms of disciplines and data exchanges. From that high-level definition, users can generate the source code skeleton required to plug the implementations of disciplines and get an actual executable model of the concept under study. Then, they are able to run numerical methods such as sensibility analysis and design of experiments in order to finally tune the model, define and run optimizations regarding given design target requirements.
\end{abstract}

\section{Introduction}

$\mathrm{O}$ NERA research engineers carry out preliminary studies on new integrated concept of vehicles (aircraft and aunchers). Such innovative vehicle design is an application field for the Multidisciplinary Design Optimization (MDO) methods: the art to optimize a set of numerical models, each representing part of the physical behavior of the overall system under study. A numerical model often relates to one engineering discipline, examples of such disciplines are: structural mechanics and mass breakdown, aerodynamics, propulsion or mission performances. Depending on the vehicle design phase, the disciplinary models fidelity may vary. At early stage, engineers use coarsed-grained models to explore the design space. Indeed, they start from the vehicle top level requirements (being a payload to transport, a range to cover, a fuel consumption target, a minimization of environmental impact, etc.) to gradually downselect a vehicle concept solution. In the way, research engineers have to craft models simulating new technologies for which no or little actual experience feedback exists and also, have to use efficient optimization techniques to encompass an ever growing number of parameters being either design variables or constraints to satisfy (Section II).

The challenge here is to come up with a collaborative design environment allowing the research scientists and engineers at ONERA to mix and match numerical models while using advanced MDO methods sometimes specifically developed to tackle the design problems at hand. The objective of such environment is to provide a shared vision of the multidisciplinary design process under construction to the team but also support software development activities and study results production. A byproduct of such environment is the capitalization of the various design processes and their results ensuring traceability, maintainability and re-usability in further studies (Section III).

By leveraging web technologies and code generation techniques, we develop WhatsOpt, a web application to support these MDO collaborative activities. Even if WhatsOpt relies largely on OpenMDAO [1] capabilities, the underlying idea is to abstract away from a given implementation by using our own model of the MDO domain (Section IV). From this model, which let us describe our multidisciplinary analyses (MDA), we can generate skeleton code (i.e. scaffolding) to ease actual analysis implementation but also process graphic representation or interface tools like optimizers or design of experiment runners to carry out our studies (Section $\nabla$ ).

\section{Motivations}

\section{A. Vehicle design and MDO tooling at ONERA}

Tackling the multidisciplinary aspects of design a novel aircraft, launcher or rotorcraft concept has been a long story at ONERA, as the Lab takes benefit from the availability of many disciplinary competences. Advances on the two legs have been made in the last decades:

\footnotetext{
* Research Engineer, Information Processing and Systems Department.

$\dagger$ Research Engineer, Information Processing and Systems Department.

$\ddagger$ Research Engineer, Information Processing and Systems Department.
} 
- On the vehicle design aspects, the collection of disciplinary models have been progressively enriched with various levels of fidelity, in order to model more and more new concepts such as Unmanned Aircraft Vehicle (UAV), reusable launcher, new configurations of transport aircraft such as Blended Wing Body (BWB), strut-braced wings, distributed propulsion or boundary layer ingestion; these studies have helped rationalizing the couplings between disciplines and the overall management of a design process,

- On the MDO tooling aspect, we first relied on commercial softwares such as ModelCenter ${ }^{\mathrm{TM}}$ to implement the multidisciplinary analyses, and concentrated the effort on the acquisition and development of skills in the field of advanced MDO methods such as surrogate modeling, single or bi-level formulations, optimization algorithms, sensitivity analyses and uncertainty management techniques.

Thanks to this sustained effort, MDO has progressively become a mainstream topic and a reference approach to conduct the integrated concept studies. Nevertheless, the tooling and capitalization issues have been partly left aside in these first steps, and leaving the process integration in the hands of commercial softwares led to large limitations : cost, lack of flexibility, difficult collaboration between distributed teams. Therefore a reboot was necessary on this aspect.

\section{B. Towards a new design environment}

In 2014 a new internal MDO project named ACADIA (standing for Design and Decision Making Workshop for Aerospace Innovation) was launched, with the aim of on the one hand continue the efforts on the methodological aspects enabling design space exploration, and on the other hand kick off a new approach regarding the integration framework, tackling the aforementioned limitations. In order to break the dilemma between efficient but costly and closed commercial softwares, and ad-hoc developments that lacked capitalization and consumed a lot of computing resources, the choice was made to rely on open-source frameworks having already a good development community. As the current most used scientific language, Python was promoted as the main wrapping tool of toolboxes and disciplinary models. In parallel, the OpenMDAO software was selected to be the basis of the multidisciplinary processes implementation. Rather than developing a full proprietary MDO framework, the aim of ACADIA was shifted to concentrate on the building blocks enabling a smart use of OpenMDAO in the ONERA context, and to ease communication between disciplinary experts, vehicle architects and MDO methods developers. As a first vision these building blocks on top of OpenMDAO were the enhancement of the datamodels used, the conversion of disciplinary and methodological expertise into toolboxes and libraries, and finally "something" being the front-end enabling an easy process building and execution.

\section{The era of extended collaboration}

In the meantime, the MDO community extended, both at local and international levels, and new needs came with this collaboration opportunities.

- With the development of the MDO topic, relationships were strengthened with universities such as ISAE-Supaero and the University of Michigan, leading to the need to share toolboxes, documentation, and teaching use-cases in the format of documented notebooks; therefore a design environment has to be easily sharable, and make efficient use of toolboxes available.

- On the European side, the MDO community structured itself under the flagship of the EC project AGILE [2] dedicated to collaborative MDO. A set of new technologies to ease process construction, communication between teams and data exchange, and results visualization are being developed, and there is a incentive for ONERA tools to interoperate with these new technologies.

- As an example of industrial involvement in the field of MDO, the MDA-MDO project at IRT St-Exupery in Toulouse promotes a new framework called GEMS [3] to tackle complex, high-fidelity use cases in the field of aircraft design; the design environment at ONERA should look for interoperability with these emerging frameworks.

As a consequence, mixing these new collaboration needs with the principles identified within the ACADIA project led to the main drivers for the WhatsOpt development. 


\section{WhatsOpt vision}

\section{A. OpenMDAO background}

Back in 2012, we sought for a way to allow research engineers to use in-house surrogate modeling methods. Surrogate methods are numerical techniques to build an approximation model of a more complex time-consuming models. They are commonly used in MDO and applied in overall vehicle design. We choose OpenMDAO 0.x as an integration framework. It had good properties regarding our requirements and we contributed to OpenMDAO by developing multi-fidelity surrogate management in the framework.

In 2015, as we enlarge our vision to encompass the building of overall vehicle design processes, NASA Glenn OpenMDAO team reboot the project based on the work done by J.Hwang during his PhD thesis at the MDOLab [4]. Still open-source, written in Python and still MDO-focused the new version 1.x was tailored to improve optimization performance through the extensive use of derivatives computation techniques. In the meantime, the abstraction layer was simplified to allow straightforward numerical models integration. In this opinionated release, the OpenMDAO team removed the GUI and automated MDO formulations.

Finally in 2017, NASA Glenn OpenMDAO team unveils a new major version 2.x of OpenMDAO [1] modifying the core but also the user-facing API (though the changes were not as thorough as in the previous release).

\section{B. WhatsOpt inception}

When we started to reflect on technical solutions, the OpenMDAO framework choice was a no-brainer for us as it appears to cover several requirements, mainly: open-source, written in Python, well-documented, dedicated to MDO (models and methods integration) and actively developed by the NASA Glenn Research Center.

While OpenMDAO covers MDO process implementation and execution, we focused our attention on the development of a team collaboration capability and user interactions to complement it. As MDO requires the simultaneous involvement of many engineers with different profiles (and computer skills), ranging from discipline specialist to optimization researcher and vehicle architect, it appears to us relevant to develop a web application to cover requirements related to information sharing, capitalization and user experience support.

The development context (time and resources) of WhatsOpt makes us value a pragmatic approach over a comprehensive design up front strategy.

As we had previous experiences with the Ruby on Rails (RoR) framework, we started using it to develop WhatsOpt. RoR framework has somehow changed the web development when it came out in 2004 (proof being numerous RoR-like frameworks were created). The framework is built upon the expressiveness of the Ruby language and enables an approach based on rapid prototyping and user feedback. Its success came also from a key principle summarized by the 'convention over configuration' motto, meaning that if you follow some well-thought rules selected by experts, things are getting a lot simpler and you can be more productive as less boilerplate code is required. Back then, this philosophy contrasts with the legacy Java/J2EE/Hibernate technologies where configuration was the cornerstone, could be complex and daunting. Since its first release, Ruby on Rails (currently version 5.2, 6.0 upcoming) has also kept up with web technology evolution and also embraces the rise of JavaScript front-end frameworks.

One can question the use of RoR as we could have used a web Python framework to keep using the same programming language. It appears that coupling between the web application WhatsOpt and OpenMDAO is not that tight (each one runs in its own OS processes) and finally our expertise in Ruby on Rails ecosystem outweights the benefits of potential Python solutions.

\section{User stories}

Following an iterative and incremental approach, we gradually developed features to allow the user to:

- manage multidisciplinary analyses:

- visualize a multidisciplinary analysis with its disciplines and variable connections,

- import a multidisciplinary analysis from a legacy in-house data format (Excel),

- export a multidisciplinary analysis as an OpenMDAO skeleton process,

- import a multidisciplinary analysis from an existing OpenMDAO code,

- edit a multidisciplinary analysis: interactive edition of disciplines and their connections and variables information,

- manage operations on multidisciplinary analyses: 
- define an optimization problem using a multidisciplinary analysis: specifying design variables, objective and constraints.

- support for remote execution of operations that use local multidisciplinary analysis: optimizer, design of experiment runner "as a service".

- support for operation results visualization : scatter plots, parallel coordinates, line chart, radar plots.

- support for operation results export as classic csv data files.

- manage notebooks: store and view Jupyter notebook (static rendering)

- manage geometric models: store and view 3D OpenVSP models

- manage access rights on his/her multidisciplinary analyses

To bridge the gap between the execution framework OpenMDAO and the web application WhatsOpt, we developed a command line tool named wop. Indeed, while the information is centralized within the web application, the MDO processes are executed in the user Python environment. The command line tool is a key element to have a seamless communication between the user local environment and the remote WhatsOpt application server. The Figure 1 shows the main components of WhatsOpt which follows a classic web application architecture.

\section{WhatsOpt server}

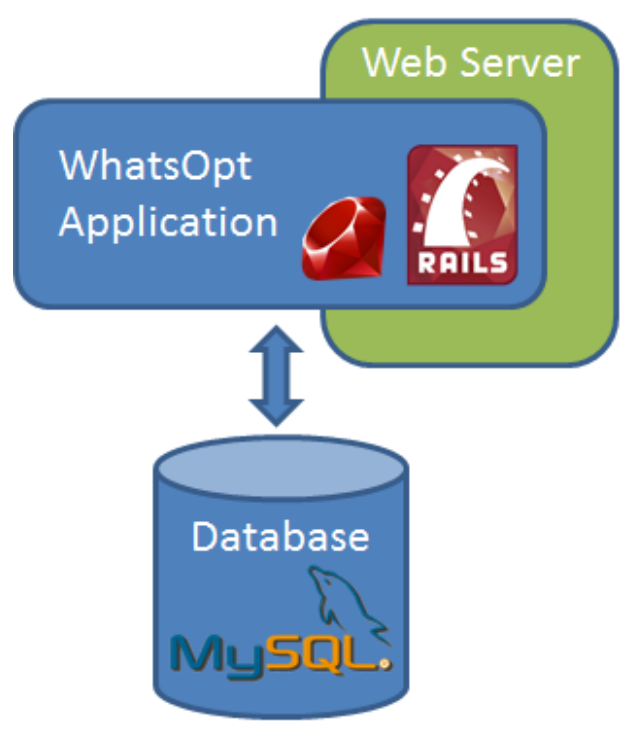

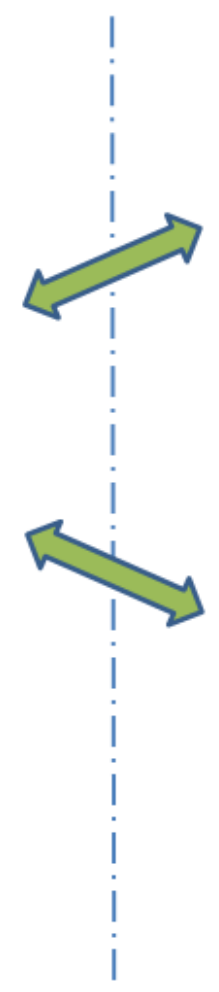

\section{User environment}

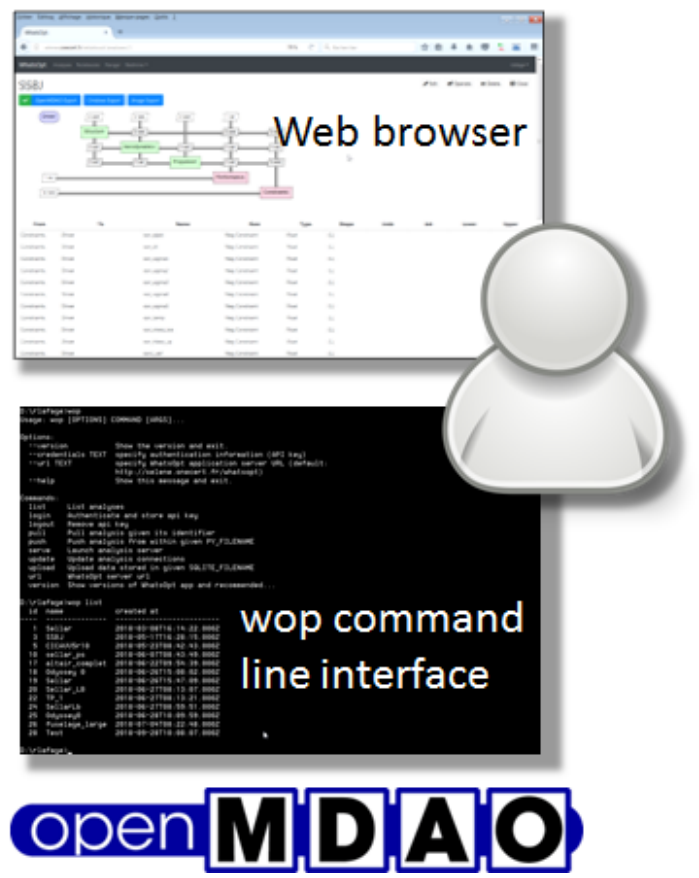

Fig. 1 WhatsOpt architecture schema

\section{WhatsOpt implementation}

\section{A. Model-based approach and code generation}

In order to isolate our MDO process developments from external contingencies, we decided to develop on top of the process execution framework our own model for defining MDO processes. Such model-based definition allow code generation and is a way to isolate our code from framework changes and ease the migration of our MDO processes from one version to another as well as the migration from a framework to another.

The usage of a model to generate code pertain to the field of Model Driven Engineering. In that context, the Object Management Group (OMG) through its standards in distributed software engineering has popularized a generic hierarchy of model abstractions: 
- M0: the actual system under study

- M1: a model of the system

- M2: a model of a language (a meta-model) to express a model of the system

- M3: and finally a meta-meta-model, the hierarchy ends here as that model can be modeled with itself.

MDE approach puts models at the core of the development of engineering systems not only using them as documentation purpose but to produce the actual implementation of the system.

The definition of the WhatsOpt model used to define MDAO processes is straightforward. As for now it is simply focused on the definition of multidisciplinary analysis (Fig. 2).

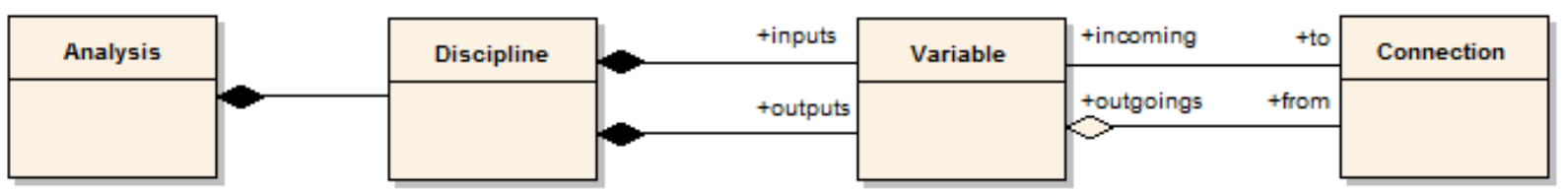

Fig. 2 WhatsOpt model for multidisciplinary analyses

Figure 3 shows the WhatsOpt model within an OMG model like hierarchy at level M2. This model can be seen as a meta-model for describing various models at level M1 expressed in projecting languages being an XDSM diagram, an analysis implemented in OpenMDAO or in RCE [5] frameworks or an analysis server implemented with Apache Thrift. Those projections are currently implemented by WhatsOpt and will be described in the following sections. In turns those models are modeling an actual aircraft considered at level M0. For completion, we have represented a meta-meta-model at level M3 which is in this case the language used to express a class diagram.
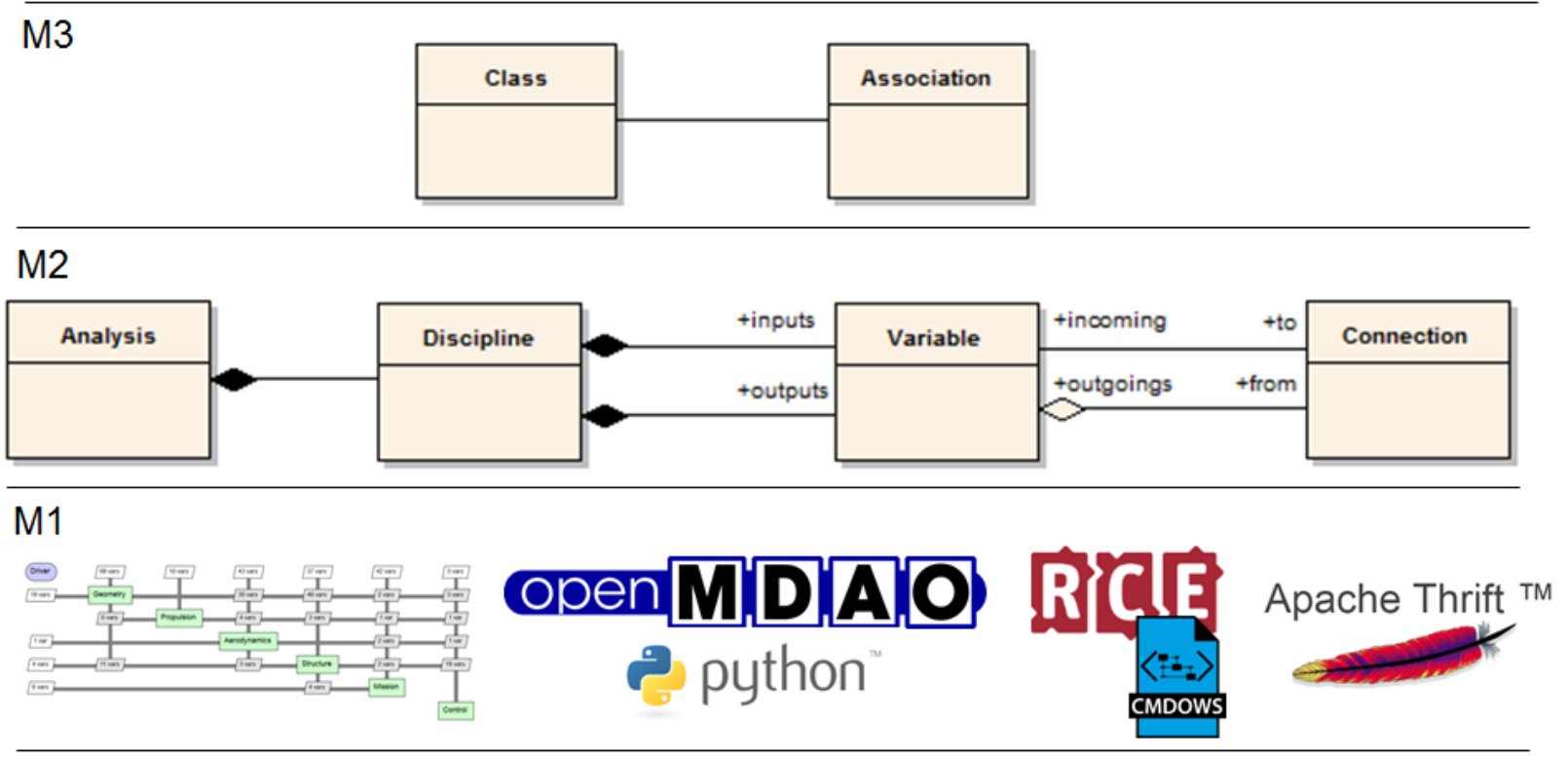

M0

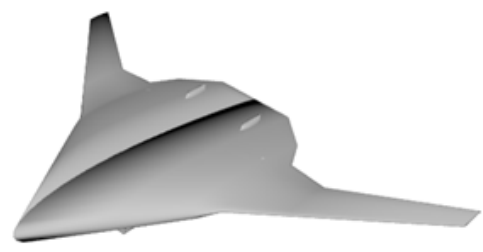

Fig. 3 WhatsOpt meta-model hierarchy 


\section{B. User stories implementation}

\section{Manage multidisciplinary analyses}

The first use case we addressed is the multidisciplinary analysis visualization. In that context, we developed a MDO process visualization capability, namely XDSMjs [6]. This visualization library is based on the graphical notation developed by Lambe and Martins called XDSM for eXtended Design Structure Matrix [7]. With the XDSM definition, they provide a corresponding LATEXpackage to publish XDSM diagrams in papers. We implemented an XDSM generator in javascript in order to be able to support the XDSM notation in a web page with the added-value to be able to interact with the diagram. This XDSMjs library is available under open-source Apache 2 license.

Since then, that library was reused in the context of the AGILE European project by RWTH Aachen University as a bootstrap for their own visualization capability VISTOMS [8] and also by the IRT Saint Exupery to visualize MDO Scenarios generated with GEMS, their MDO formulation framework [3].

To ease the migration to WhatsOpt, we develop an import feature from a legacy Excel format used by the integrated concept team. Basically, the team formalizes the definition of the process meaning: identifying disciplines, input and output variables and connections (i.e. data exchange between disciplines through variables). They already used this definition to generate skeleton code for ModelCenter ${ }^{\mathrm{TM}}$, the commercial tool used so far at ONERA to implement design processes.

Then, we developed Python code generation from the analysis to export an OpenMDAO process skeleton. This skeleton consists in OpenMDAO components built from identified disciplines. Those components are implicitly connected through variable name matches in a single root OpenMDAO group named after the analysis name (see example Figure 7 ).

Doing this, we somewhat committed to a preferred top-down approach where the user defines his or her somewhat "dreamt analysis" by defining disciplines (as simply as giving its name) and named connections between those disciplines. Then input/output variables of the connected disciplines pop up automatically from connection names and by construction have a unique name across the whole analysis. At implementation level, it allows to do automatic connection between components as soon as one component produces a variable consumed by one another.

The opposite approach would be to start with legacy tools interfaces and interconnect components by defining the connections between input and output variables with different names. This later approach is used in the AGILE project where they deal with large-scale cross-organizations MDAO processes ([2], [9]) which requires a high degree of formalization. At a smaller scale, like the one we address here, we value simpler straightforward approach based on unique variable naming. Obviously the link to legacy software has to be done later on at OpenMDAO Python wrapper level.

With this top-down approach, we implemented full creation and interactive edition of the multidisciplinary analyses. The user starts to add disciplines (i.e., a discipline name) then connections between disciplines (i.e., a comma separated list of variable names). Internally with regards to our meta-model, Discipline objects are created together with input/output Variable and Connection ones. The XDSM visualization is updated accordingly. Variable object meta-data, that is type, units, initial value can be filled gradually later on (default to float initialized to one). The Figures 4, 5.6illustrate that analysis edition on WhatsOpt.

Obviously, if other people are involved for instance because they provide a discipline implementation, they can check the technical feasibility regarding their own components. The analysis can be built interactively during dedicated meetings or people can check the status of the analysis, the process plan, and give their feedback asynchronously.

On the XDSM view (see example Figure 9), disciplines are represented by diagonal elements while variable connections are off diagonal ones. There is a first default component named Driver which is more a user interface artifact to represent the actor that uses the multidisciplinary analysis being either the user, an optimizer or a design of experiment runner. One use an analysis by providing its global inputs (which are the outputs of the Driver) and collect its global outputs (which are the inputs of the Driver). As a default, global inputs have the role of design variables and global outputs are responses of the analysis.

When the user ends analysis edition, OpenMDAO code is automatically generated on the server and run in order to detect connection errors as soon as possible. We can notice that when the analysis is created through WhatsOpt, the multidisciplinary analysis will be correct by construction. The code is runnable as all input or output variables are initialized to one. The automated run makes more sense when the analysis is created through an Excel import action as it allows to detect variables or connections mistakes by using built-in OpenMDAO checking.

At this stage, the user can export OpenMDAO source code and get the skeleton of an executable analysis. Once the code is generated, the user has to provide computation content of the various disciplines involved with regards to the 
programming interface (input and output variables) defined previously (see in Figure 7 the compute() methods of the discipline classes). The user can also adjust the solvers provided by OpenMDAO used to converge the multidisciplinary analysis.

When you start to generate code from a model, the moment comes inevitably when you want to go back from the code to the model. So, from the beginning, the idea was to enable the user to push back changes made within OpenMDAO source code back into WhatsOpt, thus enabling the round trip between WhatsOpt modeling and the actual implementation. Thanks to OpenMDAO inner model introspection we are able to create a corresponding analysis in WhatsOpt. When the legacy OpenMDAO process implementation is not trivial, this provides a easier way to grasp the implementation content by using WhatsOpt process exploration capability.

Though the need could have come up by itself in WhatsOpt, this previous capability induce the ability to display and manage nested analysis in WhatsOpt. Indeed as a WhatsOpt analysis maps to an OpenMDAO group, the ability to nest groups in OpenMDAO maps to the ability to nest analyses in WhatsOpt. The ability to nest analyses is generic and have to be implemented in WhatsOpt to be able to handle complex analyses architectures.

\section{Manage multidisciplinary operations}

Once the user has implemented disciplines, he or she wants to execute the process. So, with the code generated by WhatsOpt for the analysis itself, four scripts are provided to operate that analysis:

- run_analysis.py: simply run the analysis once with the given parameter initialization.

- run_screening . py: run the analysis to compute sensitivity analysis using the method of Morris thanks to SALib library [10].

- run_doe.py: run the analysis with regard to optimized latin hypercube design of experiment using SMT library [11].

- run_optimization.py: run the analysis to carry out an optimization of an objective (output variable) with regard to design variables (selected among input variables) subject to constraints (positive or negative inequality involving an output variable) using the Scipy SLSQP optimizer.

The first script is used merely as a debug test to ensure that the analysis can be executed properly. The three other scripts generate sqlite database (using OpenMDAO recorder capability) that can be uploaded by the user to WhatsOpt thus making a link between the multidisciplinary analysis specification and design of experiments runs or optimization results. To use these later scripts, the user has to adjust variables roles: select design variables (true variables) and parameters (fixed values) from multidisciplinary analysis inputs while optimization objective and constraints are built out of analysis outputs (see Figure 55. Finally the user can tweak the scripts to adjust the parameters of the operations.

At that point, a new idea came up to allow to move part of the execution of the operation from the user local environment onto the WhatsOpt server. In this second approach we keep generating the scripts but this time on the WhatsOpt server and just call the disciplines remotely on the user local environment where the analysis implementation is. We use the Apache Thrift Remote Procedure Call (RPC) framework [12] to call the analysis disciplines remotely.

Apache Thrift is a framework to develop cross-language interface we used on other in-house project at ONERA. Its use is a bit over-engineered at the moment as we use it for Python to Python communication (i.e. some native Python solution could have been used). But previous experience with this framework and potential future capabilities like implementing disciplines in other languages settle our choice.

Thus, together with the multidisciplinary analysis OpenMDAO code and the operation scripts, we also generate the client and server code out of the input/output disciplines specifications allowing to expose and use the disciplines' computations remotely.

From the user standpoint, in his/her local environment, he runs a run_server.py script that waits for incoming calls (namely an analysis server). Then going on a WhatsOpt dedicated page (see Figure 10, the user can specify:

- a name for the operation,

- the host where the analysis server runs,

- select an operation driver being either a single run, a DOE runner or an optimizer,

- and depending on the previous selection some parameters of the driver (e.g. a number of points in case of a design of experiment runner or the tolerance of an optimizer, etc.).

After selection, the user is able to run the operation from the WhatsOpt server which calls the analysis code in the user local environment. We display execution logs to the user and he or she is able to interrupt the operation. At the end of execution, results are automatically available in WhatsOpt (see examples Figures 8 and 13 ).

With this second approach which can be called 'Analysis operation as a service' mode, we can highlight several 
advantages:

- installation of algorithms are factorized and done once on the server. This is interesting as some optimizers compilation may be complex or not feasible in the user environment.

- the parameterization of the methods is made available through a graphical interface.

- the parameterization of the operations can be stored.

- the results are stored together with the operation execution.

The drawback of this approach is the fact that the user is somehow restricted by the parameterization available through WhatsOpt while he or she has full control if everything runs in his or her environment. A second limitation is that this feature is only available on the local network as the user host has to be reachable from the WhatsOpt server.

\section{Command line interface wop}

When it comes to go back and forth between WhatsOpt graphic interface and OpenMDAO source code, the only usage of the graphic interface is cumbersome. So it appeared very early that a command line interface to integrate smoothly between local environment and remote server would be valuable (not to say mandatory as users gain in expertise). Thus, we developed wop, the WhatsOpt command line interface.

This command uses web services exposed by WhatsOpt to support the usual use cases. The user is able to trigger the following command:

- wop list: the available multidisciplinary analyses,

- wop pull <analysis-id>: generate code from given analysis specified by its id,

- wop update: update the code to take into account analysis change on WhatsOpt,

- wop upload <sqlite filename>: upload given results supposed to be obtain by running an operation on the current analysis.

- wop serve: run the analysis Apache Thrift server to enable remote operation calls from the WhatsOpt server.

The user is authenticated by an API key which is available in his or her profile page on WhatsOpt. The wop command asks for that API key when first used and/or till a valid API key is given.

Finally, we also developed the possibility to import a multidisciplinary analysis from an OpenMDAO code through the command wop push <analysis_main.py>. While the preferred way is to update the analysis from WhatsOpt, this feature is meant to be used with a code (initially generated) that has been manually adjusted (e.g. by adding or removing disciplines or variables) and the user wants to get the changes back in WhatsOpt. For an OpenMDAO code not generated from WhatsOpt, this command works properly under the assumption that all connected variables are all uniquely named which is required with WhatsOpt. Obviously, we could generate unique names (using fully qualified names) to generate an equivalent analysis but in that case further code generation from WhatsOpt could not match the original code.

\section{Manage notebooks and geometric models}

Besides the multidisciplinary analysis, the application was also meant to share information being in the form of Jupyter notebooks or OpenVSP geometric models.

Jupyter notebooks [13] are used by ONERA research engineers so they can document results of their studies and share Python code snippets. The user is able to upload Jupyter notebook that is simply displayed as an HTML page and can be downloaded by other users.

ONERA integrated concept team uses OpenVSP [14] as a tool to manage vehicle geometries. As for notebooks, WhatsOpt allows the user to upload and share with others geometric models as OpenVSP file. Relying on OpenVSP capability, the user can view the 3D models directly on the WhatsOpt web page.

\section{Manage access rights}

Finally the web application has typical authentication, authorization feature. Regarding authentication, ONERA research engineers use their LDAP account to sign in WhatsOpt.

At the moment the authorization policy is rather simple: only the creator of an asset being an analysis, a notebook or a geometric model (i.e. the owner) has write access to it. The owner can either let his or her asset as public, meaning available for any authenticated user or restrict the access to a selection of users. 


\section{Manage OpenMDAO specifics}

While WhatsOpt model described so far is independent from the projection framework OpenMDAO, in practice, it is interesting to have finer control on the OpenMDAO generated code. This is why a specific part is dedicated to tune finer details of the code generation and really specify the projection in OpenMDAO terms. In WhatsOpt it is done on a specific page where the user can specify:

- whether the container OpenMDAO group is meant to be executed in parallel or not,

- which types of solvers and their parameterization are used,

- whether a discipline is an implicit or explicit OpenMDAO component,

- whether a discipline declares derivatives.

The default configuration is: a sequential execution, default NonlinearBlockGS and ScipyKrylov solvers and their default OpenMDAO parameterization while disciplines are explicit components with no derivative (which is more than often the case in our use cases).

The idea here is to offer sufficient level of control to allow any required specialization at Python language level. Indeed, at some point it is easier to write Python code than using a graphical interface which will not be able to handle all cases anyway.

\section{Applications}

\section{A. The Sellar problem}

WhatsOpt application is first illustrated on the Sellar problem [15]. This problem is a simple two-discipline analysis and optimization problem which is often used as a kind of 'Hello world' case for the MDO domain. The so-called disciplines, namely Disc1 and Disc2, are simple analytic expressions and basically illustrate the coupling between two numerical models. A third pseudo-discipline named Functions is dedicated to objective and constraints computations. The analysis edition and optimization problem setting is illustrated in figures 4 and 5 . Finally, on the Figure 6 below the XDSM view, the variable table lists the following information:

- discipline from: the discipline that produces the variable,

- disciplines to: the disciplines that consume the variable,

- the variable name,

- the variable role: being either Parameter, Response, Design Variable, State Variable, Min. or Max. Objective or Null or Negative constraint. By default all variables from the Driver have a Design Variable role (meaning the driver will change their value), the user can choose to keep such input variable constant by changing the role to Parameter. By default, all variables consumed by the Driver have a Response role. The user can specialize them in order to define an optimization problem by selecting a variable as a Min. or Max. Objective (Fig. 5) and some other output variables as a null constraint or a negative constraint,

- the type of the variable being Float or Integer,

- the shape of the variable being 1 (scalar) or a multidimensional shape,

- the units of the variable can be specified,

- an initial value (default to one taking into account type and shape),

- the lower and upper bounds: used only for design variables; all bounds taken together define the design space.

Once the multidisciplinary analysis is created, the user is able to generate the code through the wop pull command. It generates the following files containing corresponding classes shown on Figure 7

- disc1_base.py, disc2_base.py, functions_base.py: base classes dealing with inputs and outputs declaration of the given discipline (an OpenMDAO Component)

- disc1. py, disc2 . py, functions . py: concrete discipline classes where the user implements the given discipline outputs computations from inputs

Together with the analysis itself, the run_** py scripts and analysis server described in previous section IV.B are also generated. Subsequent analysis changes done on WhatsOpt can be taken into account in the local user environment through the wop update command (only *_base.py, run_*. py and analysis server files are overridden).

With the help of the scripts followed by an upload (via the wop upload command) of the results or by using a remote call operation to the analysis server (see Figure 107, the user generates either design of experiment or optimization results.

The Figure 8 shows optimization graphics obtained by running Scipy SLSQP optimizer. We use the powerful Plotly graphic library [16] to get result graphs: 


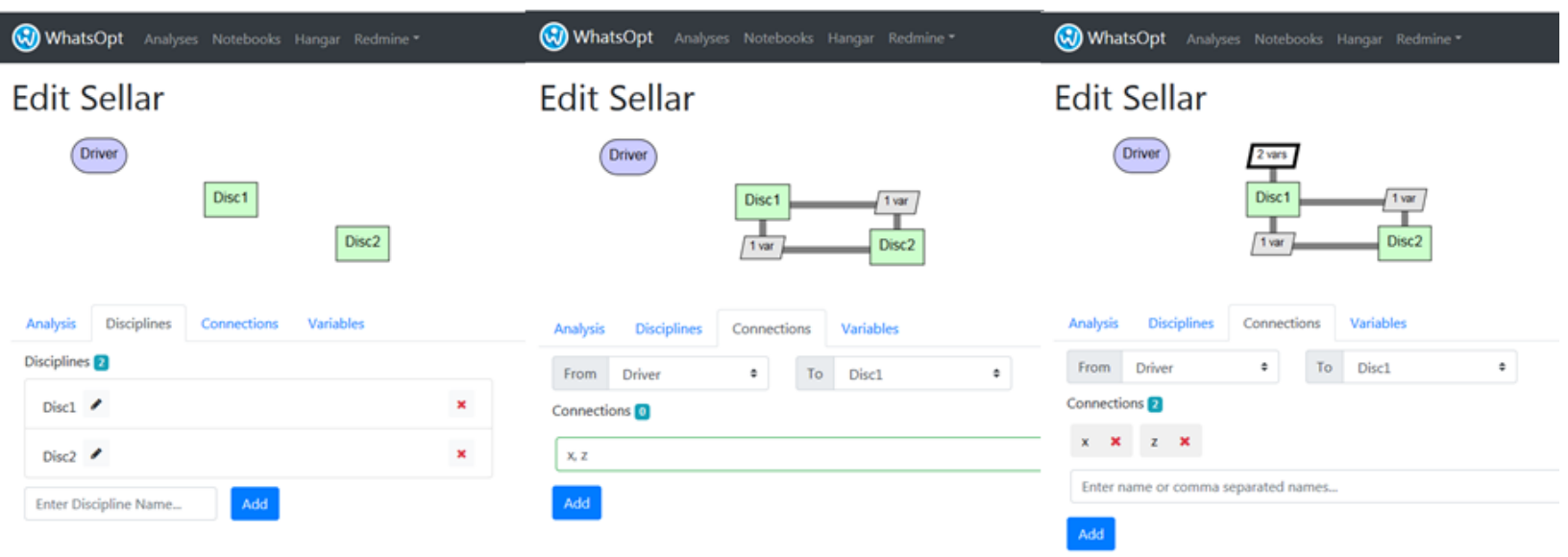

Fig. 4 Sellar analysis edition: discipline and connection creation

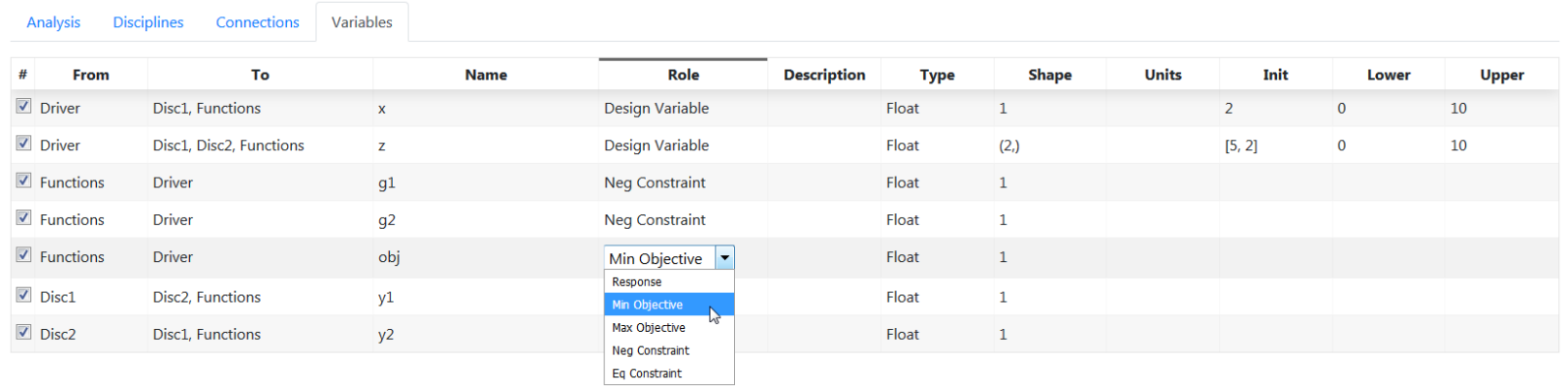

Fig. 5 Sellar optimization edition: setting 'obj' variable as minimum objective

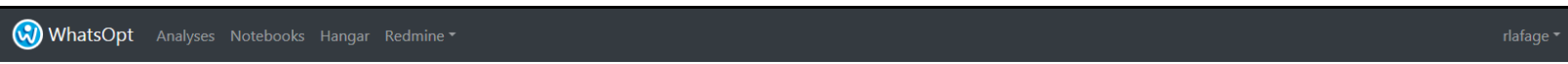

\section{Sellar} Edit Operate $\times$ Delete $\quad$ Close $\checkmark$ OpenMDAO Export Cmdows Export Image Export
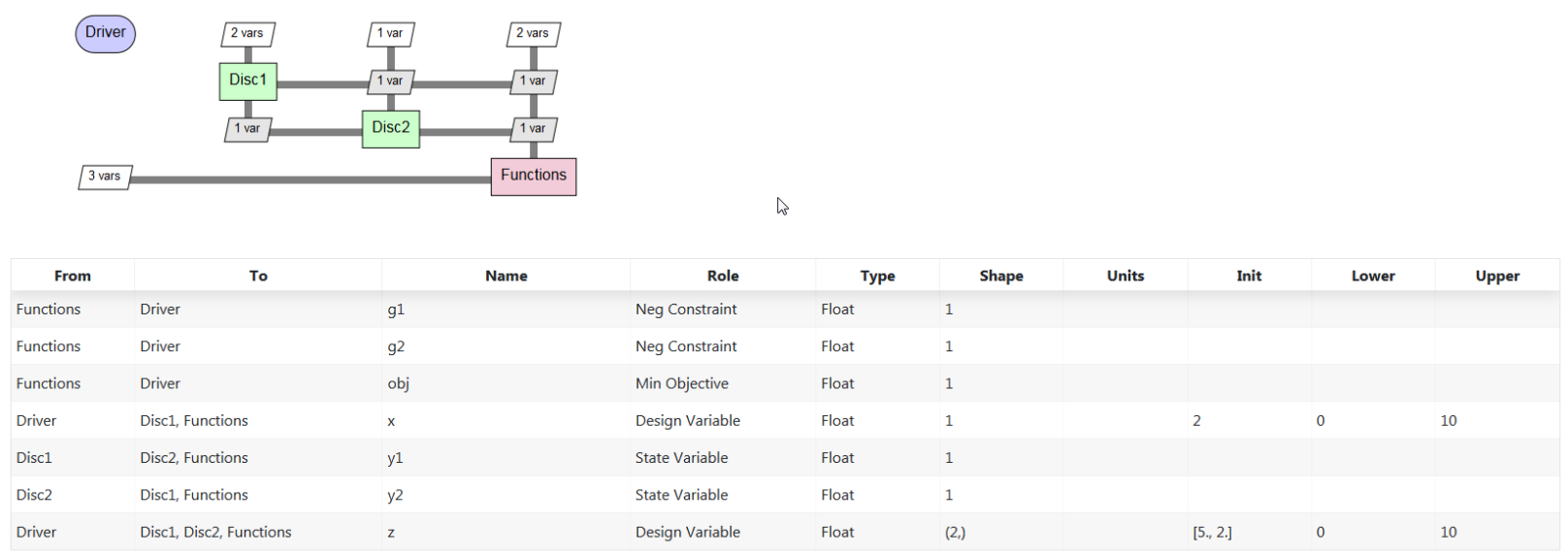

Fig. 6 Sellar analysis visualization on WhatsOpt: XDSM and variables table

- parallel coordinates: shows variables values and best candidate tuples for objective minimization are automatically highlighted,

- line chart: shows objective minimization and negative constraints along iterations (here design variables are 


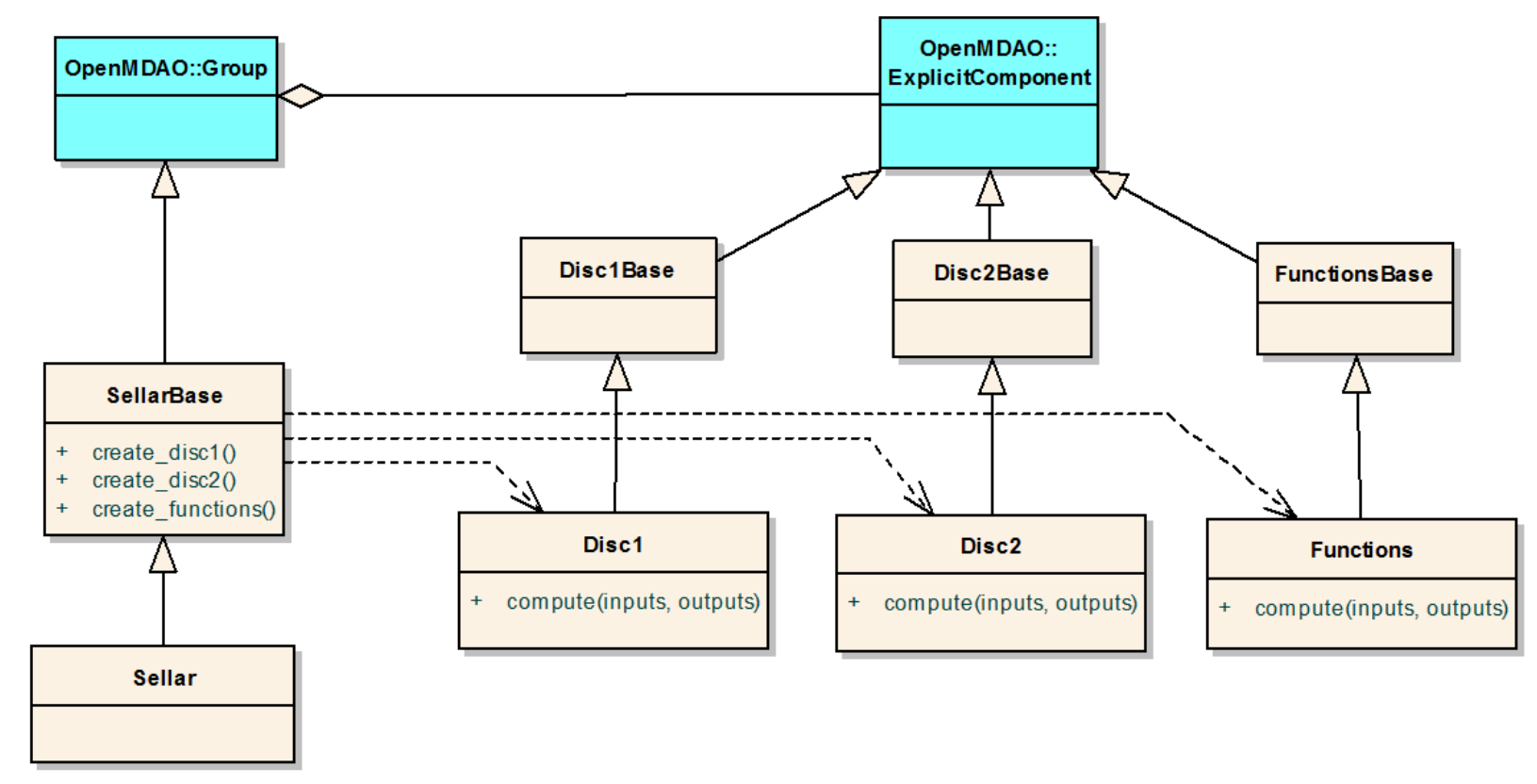

Fig. 7 Sellar generated classes example

unselected),

- radar plot: shows design variables values along iterations (here the initial iteration and the final one are shown).

\section{B. The SSBJ test case}

A more elaborated example is the SSBJ test case [17] that shows four disciplines. We have previously developed an OpenMDAO implementation solving the optimization problem which is available under open-source license [18]. As a validation exercise we pushed this implementation on WhatsOpt and we got almost immediately the analysis shown in Figure 9. We can see on this same Figure that one can select a given connection which filter the corresponding variables in the excel-like table at the bottom.

Then we pulled the OpenMDAO generated by WhatsOpt and re-wrapped the discipline computations. We could then use WhatsOpt to run design of experiment and optimization operations.

Regarding results visualization, with a growing number of variables, the user has the ability to select which ones are displayed as shown in Figure 11 .

On the Figure 12, we see some results related to a design of experiment execution on SSBJ analysis displayed as scatterplots.

On the Figure 13, we can see on the left, the mission range (R) optimization (variables are scaled) and a few negative constraints graphs. On the right, we see the design variables scaled values initialized to one and their final values displayed on a radar plot. Those results can also be exported in csv format for further analysis outside WhatsOpt.

\section{An aircraft design process: FAST OpenMDAO}

Standing for Fixed-wing Aircraft Sizing Tool, FAST is a conceptual aircraft design process jointly developed by ONERA and ISAE-SUPAERO. Its development, started in 2015, led to a modular, python, object-oriented code incorporating the basic disciplines of aircraft design (geometry, aerodynamics, propulsion, mass breakdown and balance, HQ, performance and trajectory) and implementing sizing loops to resize the aircraft upon specific Top Level Aircraft Requirements. More details can be found in [19].

From this basis, an upgraded version of FAST was developed in the frame of Alessandro Sgueglia's PhD [20], with the aim to transfer the existing process in the OpenMDAO framework, while incorporating the analytic derivatives of the whole code. The goal was to take benefit of OpenMDAO powerful numerical capabilities for optimization purpose.

In order to get proper analytic derivatives, FAST disciplines are broken down into small components almost at the 

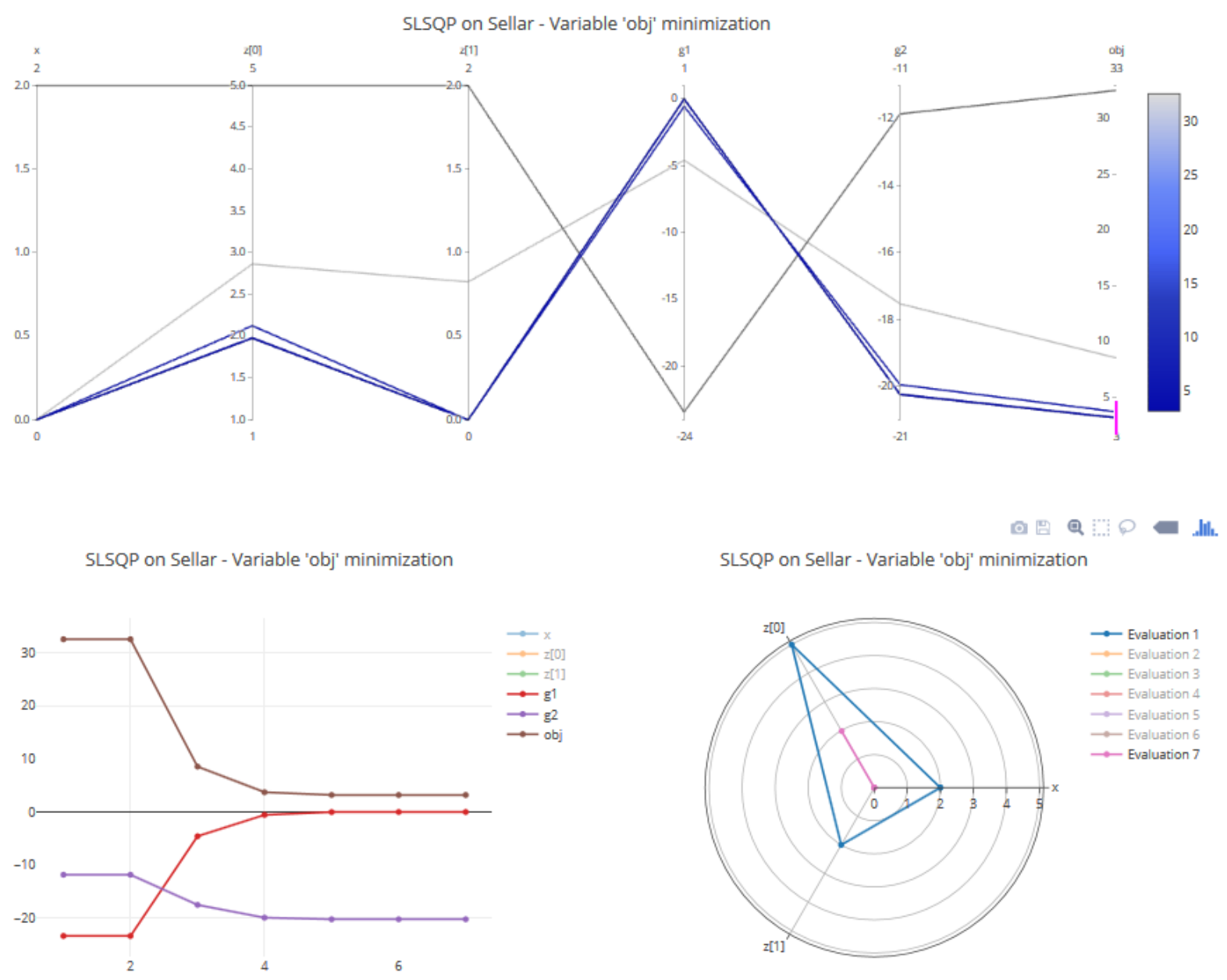

Fig. 8 Sellar optimization plots

scale of a single equation. This model development follows the OpenMDAO way as you can implement and validate simpler partial derivatives while the framework assembles them for you to get total derivatives at the end. Nonetheless this breaking down comes at the price of a complexity in terms of number of components and hierarchical structure. For the record, at the time of writing, the FAST OpenMDAO model contains 17 groups and 122 components. The Figure 14 gives an idea of the group/components hierarchy composing the FAST OpenMDAO model obtained with WhatsOpt help.

In that case, WhatsOpt acts primarily as a retro-engineering tool to help newcomers to grasp the composition of the model and explore its variables. Using the code-to-model import capability, the FAST OpenMDAO model in WhatsOpt is just one command line away. The user is then able to navigate up and down the model hierarchy by clicking respectively on sub-analyses and drivers elements in the cascading XDSMs.

\section{A Blended Wing Body study}

\section{Multidisciplinary analysis}

In 2015, ONERA began the internal reasearch CICAV project dedicated to the study of the Blended Wing Body (BWB) configurations through a multidisciplinary overall aircraft design process. The objective was to evaluate with a sufficient accuracy the expected gains brought by such a configuration, compared to the Tube and Wing derivatives. This project gathers a wide range of ONERA expertise: aerodynamics, structure, propulsion, handling qualities, aeroelasticity, acoustics and performance experts, in addition with aircraft architects, MDAO and applied mathematics experts. 


\section{(4) WhatsOpt Analyses Notebooks Hangar Redmine-}

SSBJ

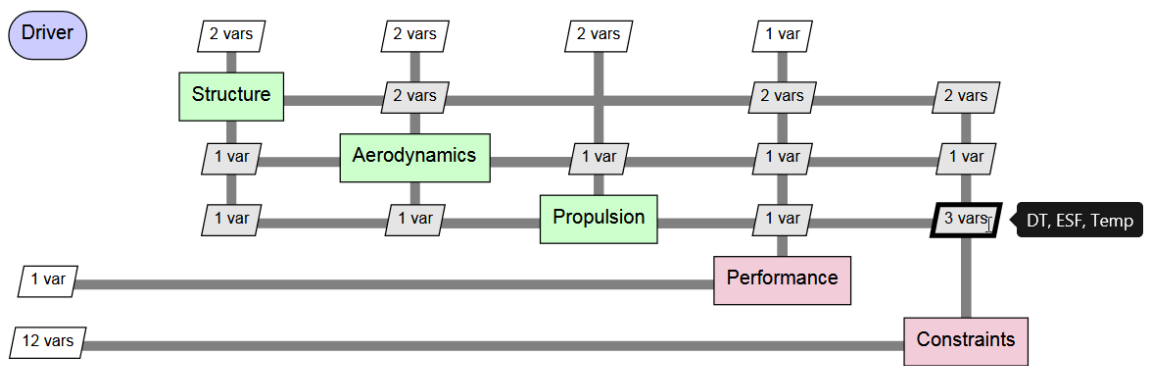

\begin{tabular}{|c|c|c|c|c|c|c|c|c|c|}
\hline From & To & Name & Role & Type & Shape & Units & Init & Lower & Upper \\
\hline Propulsion & Constraints & DT & State Variable & Float & $(1)$, & & & & \\
\hline Propulsion & Constraints & ESF & State Variable & Float & $(1)$, & & & & \\
\hline Propulsion & Constraints & Temp & State Variable & Float & $(1)$, & & & & \\
\hline
\end{tabular}

Fig. 9 SSBJ test case analysis with specific connection selection

\section{Operation on SSBJ} Specification

Operation name*
SLSQP
Analysis Server*
endymion
Driver*
RunOnce

\section{Run Abort}

\section{Status}

\section{$\checkmark$ DONE}

\section{Operation on SSBJ} Specification

\begin{tabular}{l} 
Operation name \\
\hline SLSQP \\
\hline Analysis Server* \\
\hline endymion \\
\hline Driver* \\
\hline RunOnce \\
\hline $\begin{array}{l}\text { RunOnce } \\
\text { SMT - LHS } \\
\text { Scipy - COBYLA } \\
\text { Scipy - BFGS }\end{array}$ \\
S Scipy - SISQP \\
\hline pyOptSparse - CONMIN \\
pyOptSparse - SLSQP \\
pyOptSparse - PSQP \\
pyOptSparse - NSGA2
\end{tabular}

\section{Operation on SSBJ} Specification

Operation name*
SLSQP
Analysis Server*
endymion
Driver*
Scipy - SLSQP
Objective function tolerance for stopping criterion
0.000001
$\square$ Print convergence messages
Maximum of iterations
1000

Status

Fig. 10 Operation on SSBJ test case: selection of Scipy SLSQP driver

At the beginning of the project, as no implementation was available, the CICAV team defined an "ideal process" from scratch starting with the choice of geometric parameterization and the choice of disciplinary modules to be developed. As WhatsOpt was developed at the same time, we prioritize the development of its features to support the CICAV team 
Response Variables

$\nabla$ con_dpdx $\nabla$ con_dt $\nabla$ con_sigma1 $\nabla$ con_sigma2 $\square$ con_sigma3 $\square$ con_sigma4 $\square$ con_sigma5 $\square$ con_temp $\square$ con_theta_low $\square$ con_theta_up $\square$ con1_esf $\square$ con2_esf $\nabla$ R Design Variables

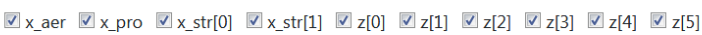

State Variables

$\square$ D $\square$ dpdx $\square$ DT $\square$ ESF $\square$ fin $\square$ L $\square$ SFC $\square$ sigma[0] $\square$ sigma[1] $\square$ sigma[2] $\square$ sigma[3] $\square$ sigma[4] $\square$ Temp $\square$ Theta $\square$ WE $\square$ WF $\square$ WT

\section{Fig. 11 SSBJ test case variable selection}

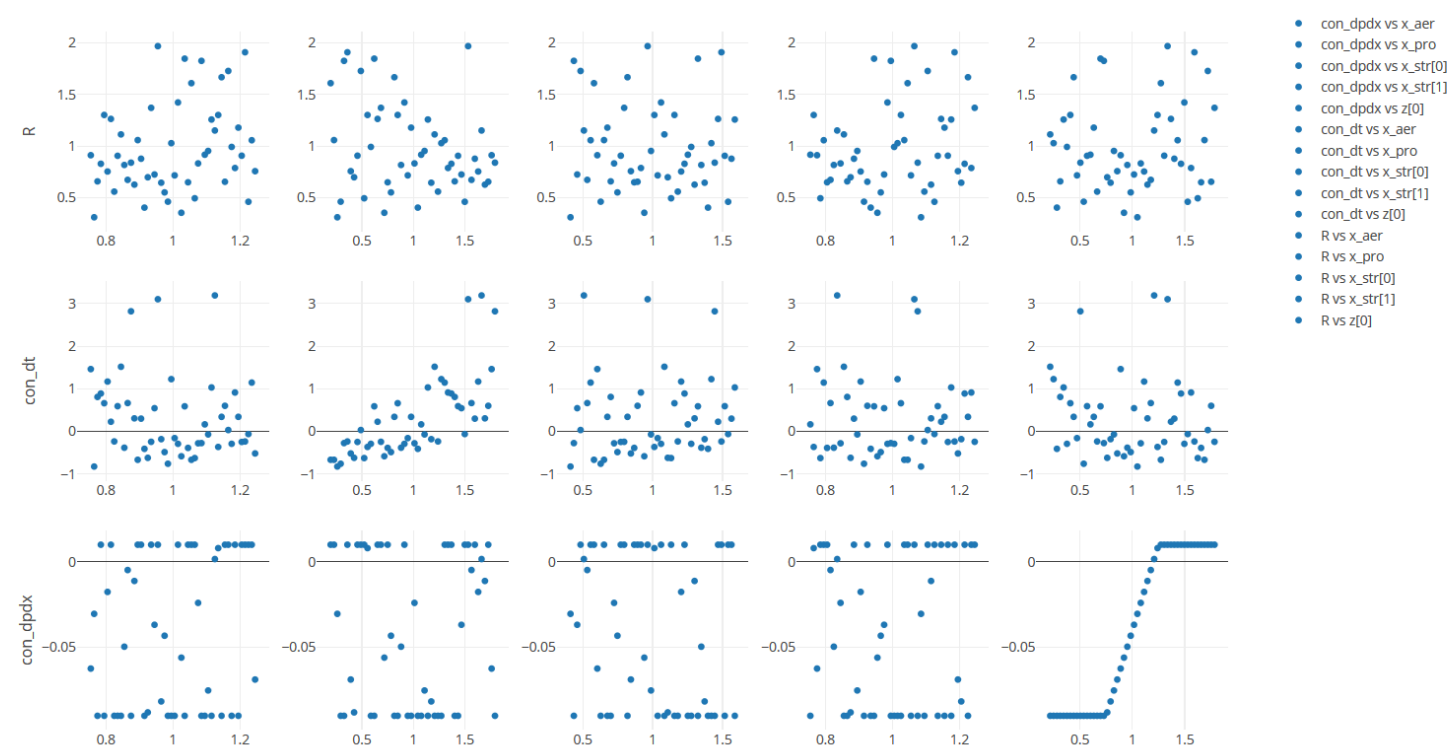

Fig. 12 SSBJ test case design of experiment results: scatter plots

work.

Initially we implemented the import capabilities, first from our in-house Excel file format then from successive OpenMDAO implementation versions, allowing to share the process structure. The Figure 15 shows various versions of the XDSM of the CICAV process imported in WhatsOpt. More details on the CICAV BWB process can be found in [21].

\section{Multidisciplinary operations}

As stated in IV.B, the workflow is exported as an OpenMDAO source code in order to obtain the skeleton of an executable analysis. As an initial OpenMDAO implementation exists, we simply wrapped the existing discipline implementations in the compute () methods of the OpenMDAO generated code allowing further developments and studies through WhatsOpt application. Thanks to run_analysis.py script, we run the MDA process and validate the behaviour of the disciplines and the overall performance of the BWB aircraft. The following step is to carry out an optimization of the aircraft configuration, the target being a long-haul commercial transport BWB aircraft, with similar top-level requirements as the A350-1000 aicraft. Without giving the details, the optimization problem is defined as follows: 

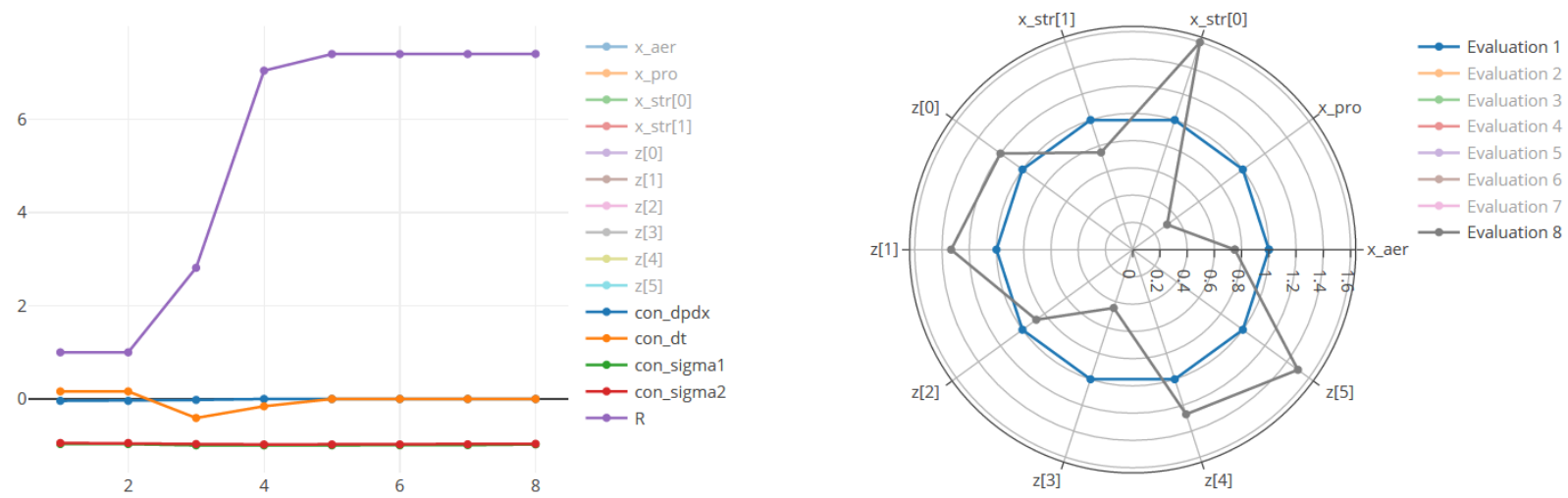

Fig. 13 SSBJ test case optimization results: line chart with the range $(R)$ maximization and negative constraints and radar plot showing initial design variables scaled values and the final ones when optimization converges

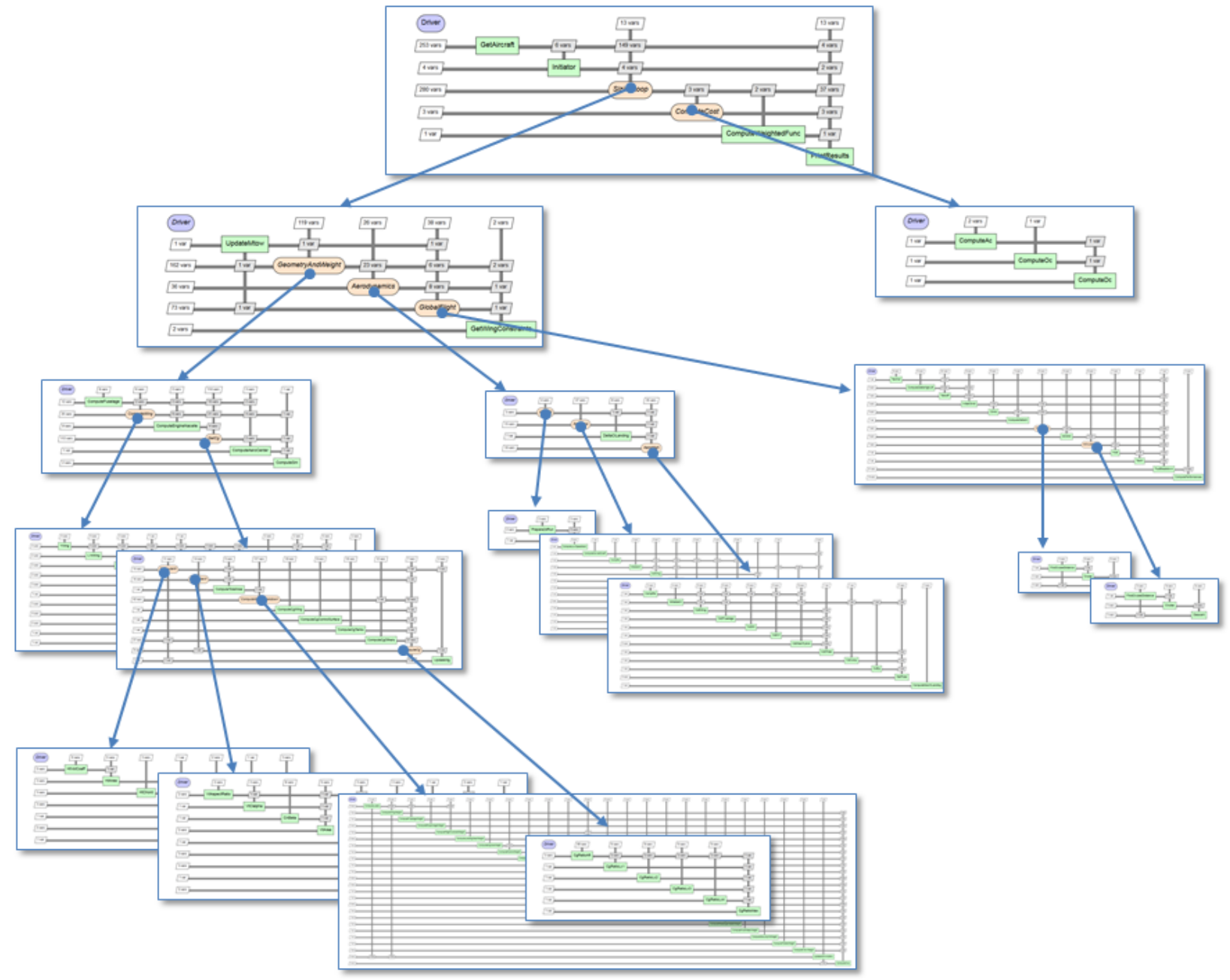

Fig. 14 FAST Openmdao model hierarchy: 17 groups and 122 components on 5 levels 


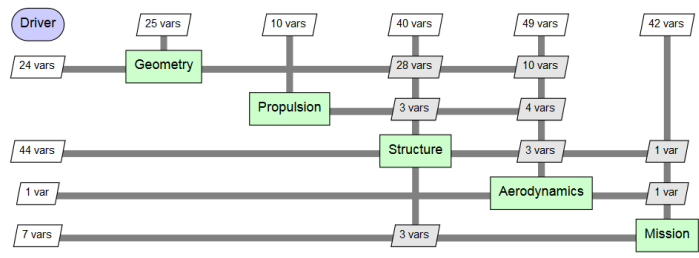

(a) Initial

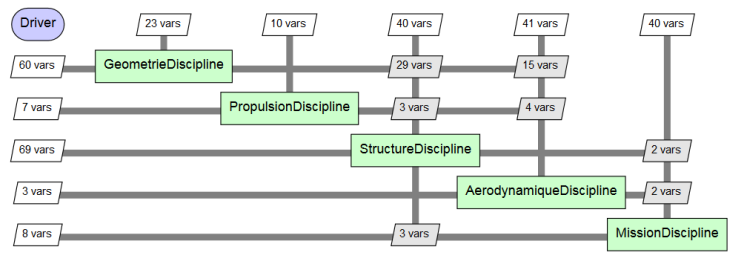

(c) Iteration 2

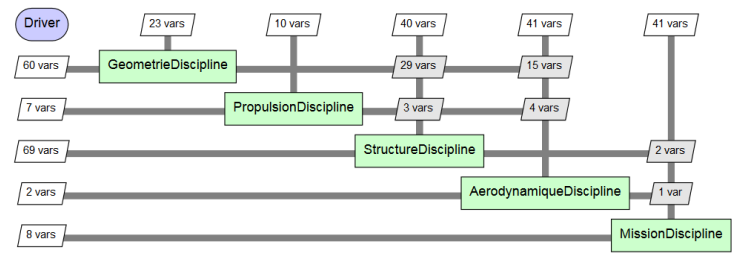

(b) Iteration 1

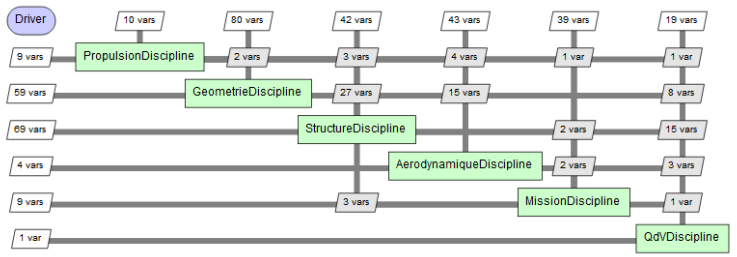

(d) Current

Fig. 15 Evolution of the CICAV multidisciplinary analysis

$$
\begin{cases}\min & \text { mission total fuel mass: } m \text { Fuel } \\ \text { w. r. t. } & 17 \text { variables ( } 14 \text { shape variables and } 3 \text { operational variables) } \\ \text { s. t. } & 3 \text { operational constraints (climb and mission duration and take off field lenght) }\end{cases}
$$

We prepare the optimization using the interactive edition capabilities of WhatsOpt. We add a new discipline Fonct0ptim dedicated to the computations of objective and constraints output variables. Once those dedicated variables created, we set their respective role (minimization objective and negative constraints in this case) and the bounds of the design variables which define the design space (see Figure [16).

\begin{tabular}{|c|c|c|c|c|c|c|c|c|}
\hline From & Name & Role & Type & Shape & Units & Init & Lower & Upper \\
\hline Driver & cruising_mach_number & Design Variable & Float & (1.) & & 0.84 & 0.75 & 0.89 \\
\hline Driver & distance_y_p1_to_y_p2 & Design Variable & Float & (1.) & & 2.5 & 1.0 & 4.0 \\
\hline Driver & distance_y_p2_to_y_p2_1 & Design Variable & Float & $(1)$, & & 6.5 & 5.0 & 11.0 \\
\hline Driver & structural_design_altitude & Design Variable & Float & (1.) & & 7620.0 & 6000.0 & 9000.0 \\
\hline Driver & top_of_climb_altitude & Design Variable & Float & (1,) & & 9448.0 & 8500 & 10750 \\
\hline Driver & vertical_surface_height & Design Variable & Float & $(1)$, & & 5.132 & 4.50 & 6.5 \\
\hline Driver & vertical_surface_sweep_leading_edge & Design Variable & Float & (1.) & & 42.0 & 35.0 & 50.0 \\
\hline Driver & vertical_surface_sweep_trailing_edge & Design Variable & Float & (1.) & & 35.0 & 30.0 & 40.0 \\
\hline Driver & vertical_surface_taper_ratio & Design Variable & Float & $(1)$, & & 0.49 & 0.4 & 0.6 \\
\hline Driver & wing_chord_p2 & Design Variable & Float & (1.) & & 14.0 & 10.0 & 18.0 \\
\hline Driver & wing_chord_p2_1 & Design Variable & Float & (1.) & & 8.0 & 5.0 & 11.0 \\
\hline Driver & wing_chord_p3 & Design Variable & Float & (1.) & & 4.0 & 2.0 & 6.0 \\
\hline Driver & wing_span & Design Variable & Float & (1.) & & 80.0 & 65.0 & 80.0 \\
\hline Driver & wing_sweep_leading_edge_po & Design Variable & Float & (1.) & & 55.0 & 45.0 & 65.0 \\
\hline Driver & wing_sweep_leading_edge_p2 & Design Variable & Float & (1.) & & 40.0 & 30.0 & 50.0 \\
\hline Driver & wing_thickness_ratio_po & Design Variable & Float & (1.) & & 0.15 & 0.12 & 0.16 \\
\hline Driver & wing_thickness_ratio_p2 & Design Variable & Float & $(1)$, & & 0.09 & 0.08 & 0.10 \\
\hline FonctOptim & Cont_climb_duration & Neg Constraint & Float & 1 & & & & \\
\hline Fonctoptim & Cont_mission_duration & Neg Constraint & Float & 1 & & & & \\
\hline Fonctoptim & Cont_take_off_distance & Neg Constraint & Float & 1 & & & & \\
\hline FonctOptim & Obj_mission_total_fuel_mass & Min Objective & Float & 1 & & & & \\
\hline
\end{tabular}

Fig. 16 CICAV optimization problem: design variables, objective and constraints definition

We generate the scripts presented in IV.B to operate the analysis. Before running the optimization, we use the run_screening. py script in order to reduce the number of design variables by identifying and ignoring (i.e. setting to 
a fixed value) the less influential ones. The Figure 17 shows the variables screening results uploaded on WhatsOpt: the sensitivity analysis of the 4 variables of interest (the objective and the three constraints) regarding the 17 design variables. A graph for a given output variable of interest shows all design variables at a location representing the effect of the design variable. The location corresponds to the mean of the effect in absolute value on the $\mathrm{x}$ axis and the standard deviation of the effect on the y axis. So, most important variables show up on the top right corner of the graph while least important ones are displayed on the bottom left corner.

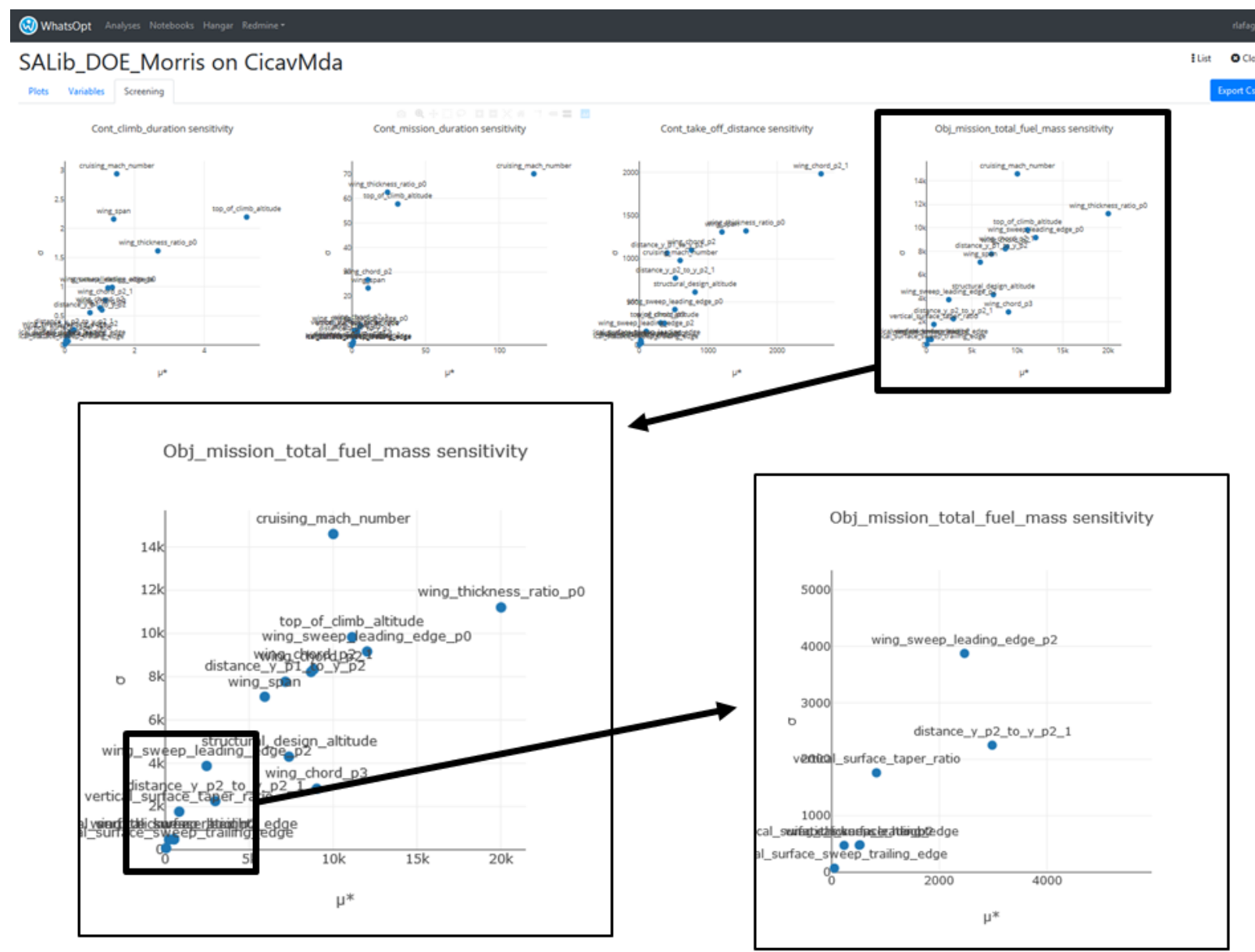

Fig. 17 CICAV variable screening: the ones with the least effect on the output variable of interest can set to a fixed value in the given optimization problem

We identify 5 geometrical variables with low effect that can be safely ignored. The optimization problem design space dimension is reduced from 17 to 12 variables. As no analytic derivative is available with the current version of the disciplines, we use our in-house gradient free optimizer SEGOMOE developed in collaboration with ISAE-SUPAERO. More details on this optimizer can be found in [22].

We run the optimization process and the results are uploaded on WhatsOpt as well. The Figure 18 gives an overview of the results visualizations. We can interactively zoom in/out, select more or less information to analyze the results. At the top, the parallel coordinate provides an overview of the behaviour of both inputs and outputs of the problem. The line chart on the bottom left presents the history of the objective function where one can identify when the algorithm changes its area of exploration with some peaks in the first half then it converges gradually stopping when the iteration budget is reached. Finally, the radar chart displays how the design variables are driven to get the final objective value.

To summarize, we took advantage of WhatsOpt capabilities to setup and operate the CICAV design process. We edited the workflow and generate automatically boilerplate code of the analysis and optimization process. We use generated scripts to explore the behaviour of the resulting model carrying out sensitivity analysis study and optimization, all along leveraging WhatsOpt visualisations. 


\section{(Q) WhatsOpt Analyses Notebooks Hangar Redminer}

Plots Variables $\quad$ Export Csv

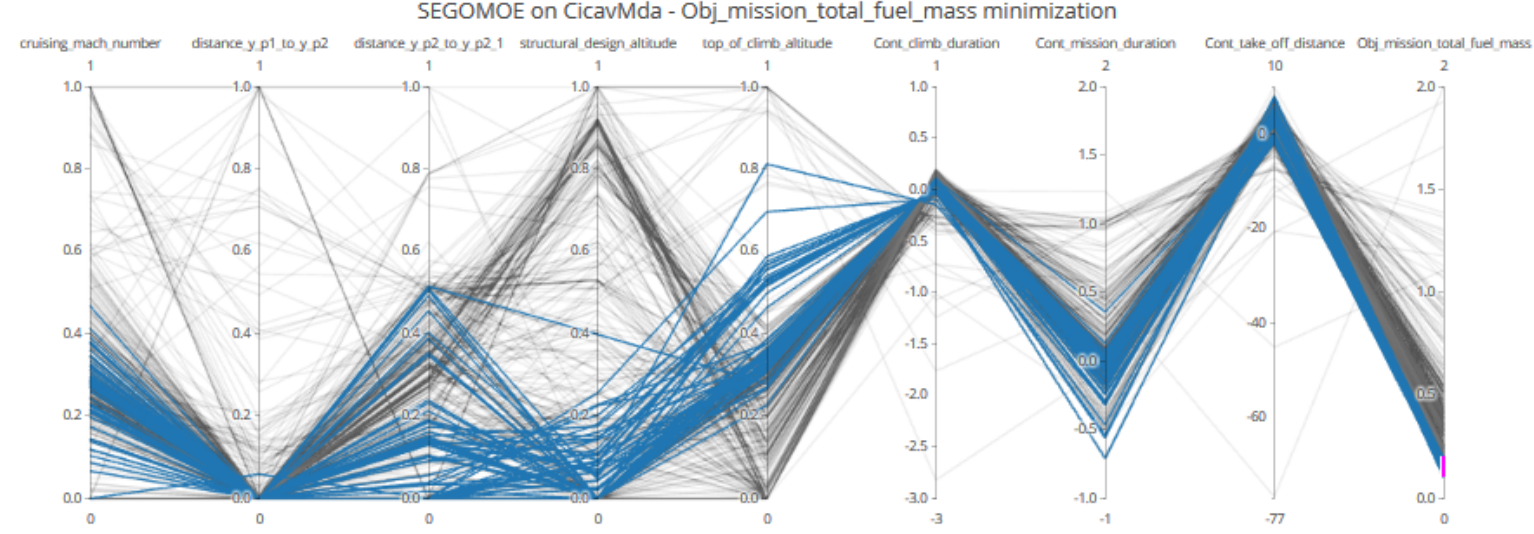

SEGOMOE on CicavMda - Obj_mission_total_fuel_mass minimization

SEGOMOE on CicavMda - Obj_mission_total_fuel_mass minimization
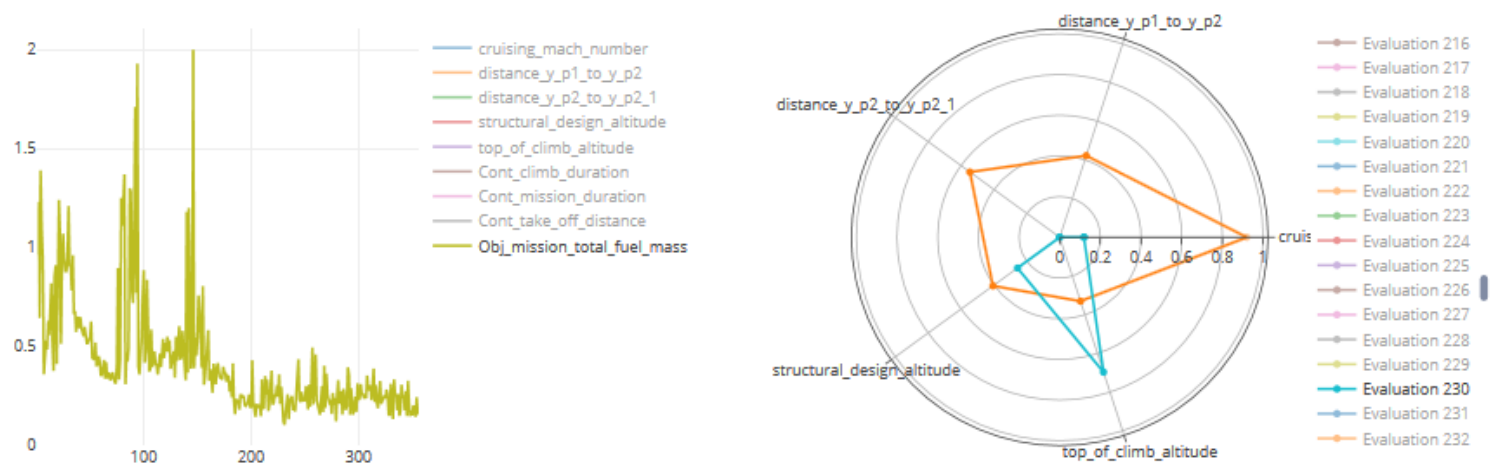

Fig. 18 CICAV optimization results : parallel coordinates (top) gives an overview of the values taken by each variables, line chart (bottom left) shows selected objective minimization over iterations while radar plot (bottom right) shows design variables scaled values at a given iteration (here the initial iteration and the 230th, the one for the minimum objective value are selected) 


\section{Conclusions and further work}

In order to help overall vehicle design team, we developed WhatsOpt, a web application supporting MDAO collaborative activities. Build on top of OpenMDAO framework, the application offers a graphical interface to edit multidisciplinary analyses and provide a support to carry out parameter studies and optimizations. It makes MDAO tools (analyses creation and operations) available for research engineers and lower the barrier for the beginners and people not familiar with MDAO tooling. It acts as a MDAO process definition and information repository for the team and finally it structures and guides actual implementation through code generation.

WhatsOpt has reached a first milestone as it is actually used for supporting studies. Its development will keep on being guided by overall vehicle design engineers needs at ONERA. Without any assumption on the priorities, we can foresee that further work will involve:

- improving analyses transformation and existing implementations reuse: the user would like to compose or merge existing analyses, but also mix and match existing implementations,

- adding surrogate modeling capability (i.e. kind of 'Surrogates a a service' feature): the user would like to create surrogate models on the server then use them in his or her MDAO processes,

- extending export capabilities: the user would like to use a MDAO framework different from OpenMDAO,

- extending MDAO processes distribution: the user would like to run disciplines distributed on different machines.

Finally, we would like to mention that we have recently deployed WhatsOpt on an online server with hopefully further demonstration and/or external collaboration [23].

\section{Acknowledgments}

We would like to thank Julie Gauvrit-Ledogar and Arnaud Tremolet for their feedback on WhatsOpt application features through the CICAV Blended Wing Body project. And we would like to acknowledge Justin S. Gray and the OpenMDAO development team from NASA Glenn Research Center as the work presented here is inspired and powered by the great framework they open-sourced.

This work was supported by two ONERA internal research projects dedicated to multidisciplinary design optimization, namely ACADIA and MUFIN.

\section{References}

[1] Gray, J. S., Hwang, J. T., Martins, J. R. R. A., Moore, K. T., and Naylor, B. A., “OpenMDAO: An Open-Source Framework for Multidisciplinary Design, Analysis, and Optimization,” Structural and Multidisciplinary Optimization, 2019. In Press.

[2] Ciampa, P. D., and Nagel, B., "The AGILE Paradigm: the next generation of collaborative MDO," 18th AIAA/ISSMO Multidisciplinary Analysis and Optimization Conference, 2017, p. 4137.

[3] Gallard, F., Vanaret, C., Guénot, D., Gachelin, V., Lafage, R., Pauwels, B., Barjhoux, P.-J., and Gazaix, A., "GEMS: A Python Library for Automation of Multidisciplinary Design Optimization Process Generation," 2018 AIAA/ASCE/AHS/ASC Structures, Structural Dynamics, and Materials Conference, 2018, p. 0657.

[4] Hwang, J. T., "A modular approach to large-scale design optimization of aerospace systems,” Ph.D. thesis, 2014.

[5] Seider, D., Litz, M., Schreiber, A., Fischer, P. M., and Gerndt, A., "Open source software framework for applications in aeronautics and space,” Aerospace Conference, 2012 IEEE, IEEE, 2012, pp. 1-11.

[6] Lafage, R., “XDSMjs,”https://github.com/OneraHub/XDSMjs 2016. Accessed: 2018-10-11.

[7] Lambe, A. B., and Martins, J. R., "Extensions to the design structure matrix for the description of multidisciplinary design, analysis, and optimization processes," Structural and Multidisciplinary Optimization, Vol. 46, No. 2, 2012, pp. $273-284$.

[8] Aigner, B., van Gent, I., La Rocca, G., Stumpf, E., and Veldhuis, L. L., "Graph-based algorithms and data-driven documents for formulation and visualization of large MDO systems," CEAS Aeronautical Journal, 2018, pp. 1-15.

[9] van Gent, I., La Rocca, G., and Veldhuis, L. L., "Composing MDAO symphonies: graph-based generation and manipulation of large multidisciplinary systems," 18th AIAA/ISSMO Multidisciplinary Analysis and Optimization Conference, 2017, p. 3663.

[10] Herman, J., and Usher, W., "SALib: An open-source Python library for Sensitivity Analysis," The Journal of Open Source Software, Vol. 2, No. 9, 2017. doi:10.21105/joss.00097, URL https://doi.org/10.21105/joss.00097. 
[11] Bouhlel, M. A., Hwang, J. T., Bartoli, N., Lafage, R., Morlier, J., and Martins, J. R. R. A., "XDSMjs," https://github . com/ OneraHub/XDSMjs 2016. Accessed: 2018-10-11.

[12] A.S.F., "Apache Thrift,"https://thrift.apache.org/ 2018. Accessed: 2018-11-03.

[13] Kluyver, T., Ragan-Kelley, B., Pérez, F., Granger, B. E., Bussonnier, M., Frederic, J., Kelley, K., Hamrick, J. B., Grout, J., Corlay, S., et al., "Jupyter Notebooks-a publishing format for reproducible computational workflows.” ELPUB, 2016, pp. 87-90.

[14] McDonald, R. A., “Advanced Modeling in OpenVSP," 16th AIAA Aviation Technology, Integration, and Operations Conference, 2016, p. 3282.

[15] Sellar, R., Batill, S., and Renaud, J., "Response surface based, concurrent subspace optimization for multidisciplinary system design," 34th Aerospace Sciences Meeting and Exhibit, 1996, p. 714.

[16] Johnson, A., "Plotly," https://github.com/plotly/plotly.js 2018. Accessed: 2018-11-05.

[17] Sobieszczanski-Sobieski Jaroslaw, S. A. J., and R. Sandusky Robert, J., "Bi-Level Integrated System Synthesis(BLISS),” Tech. rep. tm-1998-208715, NASA, Langley Research Center, 1998.

[18] Lafage, R., Dubreuil, S., and van Gent, I., "SSBJ-OpenMDAO," https://github.com/OneraHub/SSB]-0penmdao 2016. Accessed: 2018-10-11.

[19] Schmollgruber, P., Bartoli, N., Bedouet, J., Defoort, S., Gourinat, Y., Benard, E., Lafage, R., and Sgueglia, A., "Use of a Certification Constraints Module for Aircraft Design Activities," 17th AIAA Aviation Technology, Integration, and Operations Conference, 2017.

[20] Sgueglia, A., and al., "Development of a multidisciplinary design optimization framework with gradient calculation applied to hybrid aircraf," AIAA Journal of Aircraft (submitted), 2019.

[21] Gauvrit-Ledogar, J., Defoort, S., Tremolet, A., and Morel, F., "Multidisciplinary Overall Aircraft Design Process Dedicated to Blended Wing Body Configurations,” 2018 Aviation Technology, Integration, and Operations Conference, 2018, p. 3025.

[22] Bartoli, N., Lefebvre, T., Dubreuil, S., Olivanti, R., Priem, R., Bons, N., Martins, J. R. R. A., and Morlier, J., "Adaptive modeling strategy for constrained global optimization with application to aerodynamic wing design," Aerospace Science and Technology, 2019. (In press).

[23] Lafage, R., "WhatsOpt,”https://ether . onera.fr/whatsopt, 2019. Accessed: 2019-05-13. 